\title{
Cloud Platform based Software Marketing Strategy Using SWOT and Case Analysis
}

\author{
Wonju Park ${ }^{1}$ and Kwang-Kyu Seo ${ }^{2}$ \\ ${ }^{1}$ (Ph.D Student) 03016 Dept. of Management Engineering, Graduate School, \\ Sangmyung Univ., Seoul, Korea \\ ${ }_{2}^{2}$ (Professor, Corresponding Author) 31066 Dept. Management Engineering, \\ Sangmyung Univ., Cheonan, Chungnam, Korea \\ ${ }^{1}$ whatwillbe@wonjuzzang.com, ${ }^{2}$ kwangkyu@smu.ac.kr
}

\begin{abstract}
Cloud technology has changed the distribution method to software development and distribution, and not only its own cloud services and solutions provided by cloud service providers, but also the services registered by the developers through the marketplace for each service provider can do. Based on the convenient advantages of the cloud marketplace, many cloud-based software vendors are expected to grow their domestic and international markets. Many cloud service providers (CSPS) have the convenience of registering and deploying their software to the marketplace, billing and technical support to attract customers for their services and continually improve their stable sales. I'm making a lot of effort to this study analyzes the optimal cloud platform based on software marketing strategy for domestic and foreign cloud market changes.
\end{abstract}

Keywords: Cloud, CSP, ISV, Marketplace, SaaS

\section{Introduction}

Cloud service providers offer their services and solutions on-demand. In addition, we offer a variety of services and solutions that can be provided with such services and solutions through the marketplace. Marketplace provides services and solutions optimized for each cloud service provider's environment, and technical support and payment for installation and operation are also integrated through the cloud service provider.

\section{Research background}

Cloud-based software can be used on multiple platforms, regardless of manufacturer or hardware, and currently has 7,200 software and solutions listed in its marketplace based on Amazon Web Services. Domestic cloud service providers are also providing a number of solutions and services to their cloud service users through the marketplace. The sales structure method based on this market play has many advantages, and there are disadvantages to be considered. On the contrary, this researcher should consider the company developing software to increase sales through such market place. We want to help you establish the issues and business model.

Article history:

Received (March 25, 2020), Review Result (April 30, 2020), Accepted (May 2, 2020) 
The cloud market today is worth $\$ 21.4$ billion (214 billion), Gartner predicts Gartner [1]. This growth in the cloud market is not only critical to the cloud infrastructure, but also to the softways and apps that run on it, and to partner ecosystems that generate revenue and create customers.

\section{Scope and definition of terms of the study}

The scope of this study is to analyze the procedures and policies for independent software developers who develop IaaS-based services to register in the marketplaces provided by cloud service providers at home and abroad. We present the service items to be offered and how to increase sales through the marketplace utilization model.

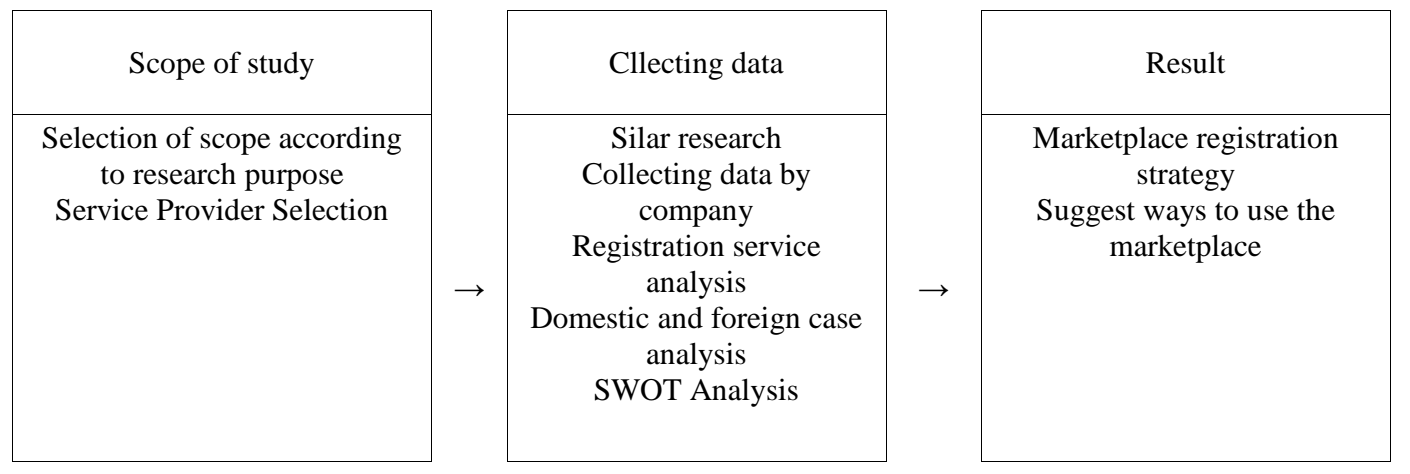

Figure 1. Research process

Providing resources for virtualized computers is called cloud computing. All processes of storing and processing data on a virtualized computer connected to the Internet, rather than on a physical computer, as a service for Internet-based computing, are provided by user requests (On-Demand), provided in various forms of service, as well as user information stored anywhere in the world over the network, and charged as much as it has been used.

The cloud is divided into three categories depending on the type of service.

Infrastructure as a service (IaaS), Software as a service (SaaS), and Platform as a service (PaaS) are divided into categories.

On the other hand, it is divided back into Private Cloud, Public Cloud, and Hybrid Cloud, depending on how the service is delivered [2].

The definitions of this distinction are divided by the form and manner provided to the user, providing computing resources such as CPU, memory, network, disk, OS, etc. according to the functional elements required by the user, and providing these resources as a service through virtualization technology in SaaS form. Application developers are provided with tools for development and elements necessary for distribution and payment in the form of a platform, which PaaS is divided into providing these platforms as a service.

The cloud gives businesses and business Provide an environment to connect. IT in cloud services Application application developed through cloud beyond technologySean, an environment that can distribute Software as a service to provide.[3]

\section{Domestic and global cloud service marketplace survey}

This section examines both domestic and global cloud market places. Through this process, the characteristics and pros and cons of local and foreign cloud marketplaces are identified. 


\subsection{KT Cloud}

As a major cloud service provider in Korea, 65 services and solutions are provided in the following category through its own marketplace [4].

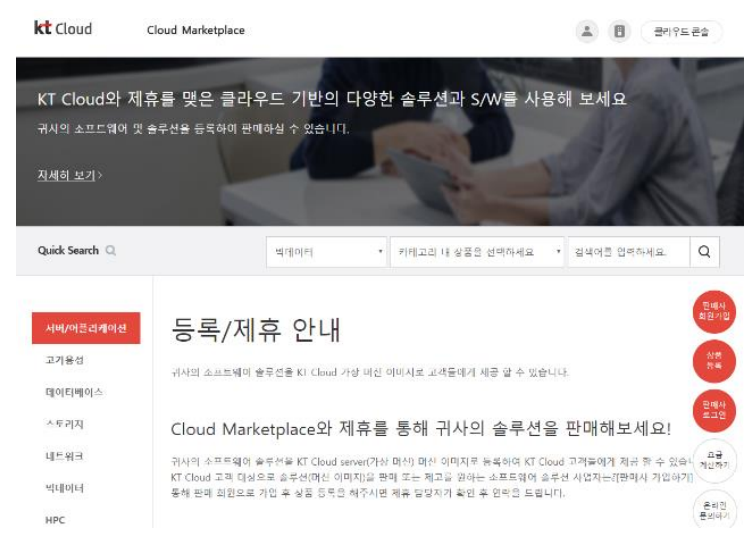

Figure 2. KT cloud marketplace registration guide

\subsection{Naver cloud platform}

As a domestic cloud service provider, it provides its customers with a marketplace classified by software type. As a registration standard, it should operate normally on Naver Cloud platform, its cloud service, and has a policy of restricting registration for multiple solutions / services with the same function [5].

\subsection{Amazon Web Services (AWS)}

It is a major overseas cloud service provider and the world's No.1 public cloud service provider. A total of 7213 services / solutions are registered in 7 categories in the marketplace [6].

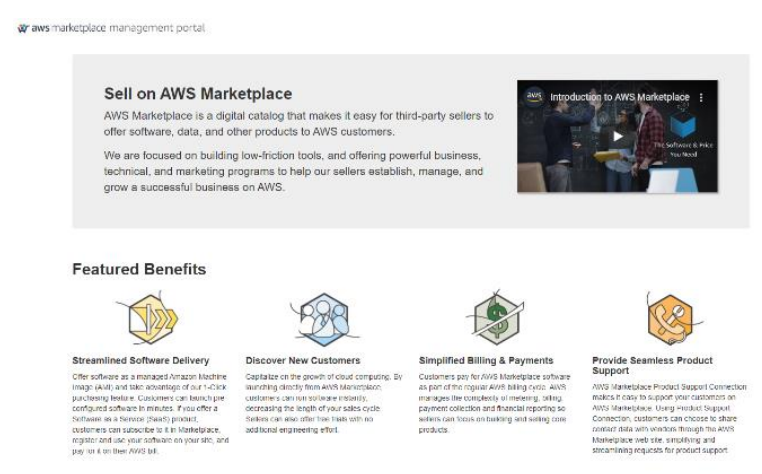

Figure 3. AWS marketplace registration guide

Marketplaces provided by loud service providers can register services / solutions developed in a form that is tailored to the needs of each service provider. It is divided into two ways and can be registered as a virtual server image form and as a service type software (SaaS). 
(1) Image upload method

The cloud service provider converts the image into a machine image that meets the requirements and policies, registers it on the marketplace, categorizes the details and technical support items, and enters the user through a dedicated portal for each cloud service provider. The registration process can be registered directly or through a person in charge [7][8][9][10].

(2) SaaS/Container upload method

Amazon Web Services provides SaaS / container software registration. In order to register the service, a separate developmental element is required to separately register the price policy, form an API for communication with AWS functions, and configure identifier registration and registration token required for the payment process.

\section{SWOT analysis and cloud platform based on marketplace strategy}

We performed the SWOT analysis of marketplace by cloud service provider. We classified the cloud platform based on marketplace of each operator by establishing the items that can be the criteria for each factor, and derive the development strategy for each factor.

Based on the SWOT analysis results, which was built earlier, each cloud service provider analyzes and analyzes each of the cloud service providers to compensate for weak internal environmental factors and strengthen the strengths. We apply the strategy of maximizing opportunity while eliminating the external environment as a threat.

In addition, priorities are set for each combination of strategies, and are used as a basis for selecting each cloud marketplace. In the mid- to long-term, the case where a developer's services and solutions are not competitive due to the basic provision of services and solutions internalized by each cloud service provider should be considered.

Table 1. SWOT analysis results of cloud service marketplace

\begin{tabular}{|c|c|c|}
\hline Strength & \multicolumn{2}{|c|}{ Weaknesses } \\
\hline $\begin{array}{l}\text { Secure abundant product / customer case (number of } \\
\text { category registration) } \\
\text { Partner ecosystem (number of registered partners) }\end{array}$ & \multicolumn{2}{|c|}{$\begin{array}{c}\text { Reduction of construction cost } \\
\text { Restriction on the presence or absence of area (lack } \\
\text { of overseas service area) }\end{array}$} \\
\hline Opportunity & \multicolumn{2}{|c|}{ Treats } \\
\hline $\begin{array}{l}\text { Overseas / Regional Specialized Infrastructure } \\
\text { Opportunity to introduce specialized business groups } \\
\text { (finance / medical / public) }\end{array}$ & \multicolumn{2}{|c|}{$\begin{array}{l}\text { Risk to Regional Regulations } \\
\text { Service Stability (Security / Infrastructure) }\end{array}$} \\
\hline External Environment Internal Environment & Strength $(S)$ & Weaknesses(W) \\
\hline Opportunity $(\mathrm{O})$ & SO Strategy & WO Strategy \\
\hline Treats(T) & ST Strategy & WO Strategy \\
\hline
\end{tabular}


Table 2. Establishment strategy for each item

\begin{tabular}{|c|c|}
\hline Division & Strategy \\
\hline SO Strategy & Strategies to take advantage of opportunities with strengths \\
\hline WO Strategy & Strategies to complement weaknesses and take advantage of opportunities \\
\hline ST Strategy & Strategies to minimize threats with strengths \\
\hline WT Strategy & Strategies to compensate for weaknesses and minimize threats \\
\hline
\end{tabular}

In future studies, the final importance of assessment factors for each SWOT group is determined through AHP techniques. Once the weight of these valuation factors is determined, we intend to derive marketing strategies using cloud platform-based marketplaces by reflecting the final weight and priority results of the sub-factors of each group.

\section{Conclusion}

Cloud computing has changed the way it is deployed from software development to deployment. In addition to its own cloud services and solutions provided by cloud service providers (CSPs), software developers can deploy registered services through cloud marketplaces [8][11].

This study proposed an optimal cloud platform-based software marketing strategy through case analysis and SWOT analysis of domestic and international cloud platforms. To this end, domestic and foreign platform-based market places were investigated and their characteristics and strengths and weaknesses were analyzed. SWOT analysis was performed using case studies and analysis results and the framework for marketing methods based on SWOT analysis was proposed. In the future, the weight of factors resulting from SWOT analysis results through AHP techniques will be determined and marketing methods based on weights will be studied for cloud platform-based market places.

Through this study, we hope to help software developers and cloud service developers such as SaaS develop marketing and market utilization plans using cloud platforms.

\section{References}

[1] Forecast: Public Cloud Services, Worldwide, 2016-2022, 4Q18 Update, Gartner

[2] Kim K.H. and S.B. Lee, "Cloud is real, no more hypothetical," Kyobo Research Report, (2015)

[3] Jeong Hyun-suk. "A study on the use of cloud computing in the 4th industrial revolution," Journal of the Korea Telecommunications Society, vol.44, no.6, pp.1213-1222, (2019)

[4] https://cloud.kt.com/portal/ktcloudportal.epc.productintro.ucloud_server_image.whole.html

[5] https://www.ncloud.com/marketplace

[6] https://aws.amazon.com/marketplace/management/tour/

[7] https://docs.aws.amazon.com/marketplace/latest/userguide/private-images.html 
[8] Andreas Menychtas, "A business resolution engine for cloud marketplaces," 2011 IEEE Third International Conference on Cloud Computing Technology and Science, (2011)

[9] Hyung Rim Choi, Doo-hwan Kim, Min Je Cho, Kangbae Lee, "The Fourth Industrial Revolution and the Countermeasures of the Marine Port Logistics Industry", The Journal of Korean Institute of Communications and Information Sciences '18-09, vol.43, no.09, Seoul, Korea, (2018)

[10] Sanghyun Kim and Hyunsun Park. Relationships with vendor dependence and organizational cloud computing expectations. Business Administration Research, vol.47, no.5, pp.1021-1047, (2018)

[11] SWOT: The Analysis of Strengths, Weaknesses, Opportunities, and Threats Excellence Marketing for Customer, pp.55-61, Korea Marketing Association, (1999)

\section{Authors}

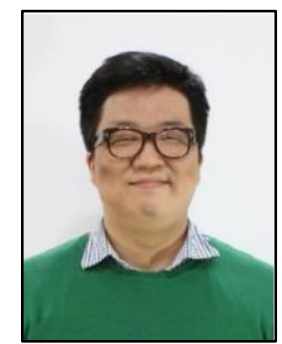

\section{Wonju Park}

Ph.D Student, 03016 Dept. of Management Engineering, Graduate School, Sangmyung Univ., Seoul, Korea

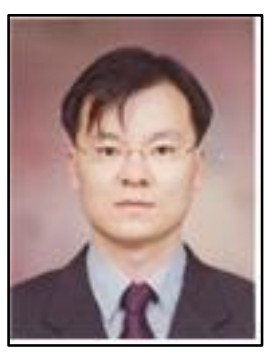

\section{Kwang-Kyu Seo}

He is a professor of Management Engineering at Sangmyung University. Prof. Seo received his Ph.D. degree in industrial engineering from Korea University. His recent research interests are in cloud computing, artificial intelligence, management information system, ICT convergence and so on. 